\title{
Detection of rebar corrosion in bridge deck by using GPR
}

\author{
Tahar Bachiri ${ }^{1}$, Abdellatif Khamlichi ${ }^{2, a}$ and Mohammed Bezzazi ${ }^{1}$ \\ ${ }^{1}$ Department of Physics, Faculty of Sciences and Technology at Tangier, University Abdelmalek Essaadi, Tangier 93001, Morocco \\ ${ }^{2}$ Department STIC, National School of Applied Sciences at Tetouan, University Abdelmalek Essaadi, Tetouan 93030, Morocco
}

\begin{abstract}
Ground Penetrating Radar based inspection technique is currently used for mapping bridge deck in order to monitor the health of this vital structure. Research over recent years in this field has focused on identification of rebar corrosion which is known to cause staged deficiencies by reducing bridge deck strength to critical levels. It is expected that using high-frequency antennas could improve discrimination of anomalies and enhance detection of small corrosion defects affecting bridge deck rebars. In this work, simulation of the input reflection coefficient of reinforced concrete bridge deck as modified by corrosion of a steel rebar is performed. The objective is to analyse the effect of the work frequency and corrosion layer thickness on the input reflection coefficient. Simulations have been carried out for various situations involving intact and corroded zones of a rebar buried in a reinforced concrete plate with the whole infrastructure overlaid by an asphalt layer. The obtained results have shown that the value of work frequency is very important and should be carefully selected.
\end{abstract}

\section{Introduction}

Ground Penetrating Radar (GPR) utilizes the characteristics of an electromagnetic field in the time domain and samples the backscattered echoes from anomalies that can be present in the host medium. GPR is nowadays used in different applications such as NonDestructive Testing based techniques for inspection of civil engineering infrastructures [1].

Principle current applications of GPR in the field of civil engineering include determination of construction features, estimation of element thickness, estimation of the concrete cover depth, location of reinforcing bars and metallic ducts, location of corrosion variations, localization of voids and estimation of rebar size [2,3].

GPR has been used since the 1970's to inspect various forms of civil infrastructures, and seems to have nowadays the widest appeal in bridge deck inspection. This method is in competition with infrared thermography which has been in use since the late 1970's and which became commercially viable two decades ago. Following recent improvements in testing equipments, infrared thermography has turned out to be interesting as a bridge deck inspection tool. However, this technique still suffers from its inability to infer the depth of a delaminated or deteriorated area.

GPR systems record the response to a radar impulse or a series of radar impulses incident on the surface of the test part. The acquired reflection echoes are processed to obtain a radargram either in the form of Ascan, B-scan or C-scan. Careful assessment of the GPR responses can often yield information about the material properties and its condition. Although anomalies can be evident in the GPR scanned image of an object, their quantification and interpretation in terms of position and main features is an issue that remains today quite confusing. As a result, all civil infrastructure defects cannot be diagnosed reliably with a single sensing modality, and inspections can just identify problems once they have progressed to a certain grade of severity.

Studying the waveforms and patterns generated during GPR scanning in terms of radargram forms constitutes the key approach towards interpretation of results. These can be presented in a variety of ways including A-scan which shows the reflected signal waveform as function of two flight travel time. Reflected signal peaks by anomalies are then quite visible on the obtained scaled curve. Associating these traces to the characteristics of anomalies is still a huge challenge that necessitates considering in general solution of an inverse problem.

The primary cause of deterioration in bridge decks is due to concrete cracking which frequently results in delamination like defects. Corrosion of steel reinforcements over time constitutes also a major concern in bridges. This can be accelerated by de-icing salts and water infiltration. Furthermore, when the reinforcements corrode they expand and a horizontal crack is formed in the reinforcement level [4]. Most of applications have focused on detecting corrosion induced deteriorated areas in bridge decks [5].

Condition assessment and system management of Bridge decks have been carried out by using GPR for quantifying concrete overlay above reinforcing steel, pavement thickness, and concrete permittivity to assess concrete quality. GPR units were also found to be

\footnotetext{
${ }^{a}$ Corresponding author: khamlichi7@,yahoo.es
} 
capable of identifying concrete with high chloride and moisture contents since these factors produce high variable reflections of the radar wave at the overlay-deck interface [7]. Changes of the wave reflected by concreterebar interfaces have been used to identify rebar characteristics and to assess corrosion probability [8]. Bridge decks overlaid with asphalt do not pose a problem for GPR mapping of underlying reinforced concrete infrastructure since asphalt consists principally of aggregates that behave as a dielectric medium for electromagnetic wave propagation.

Both ground-coupled and air-coupled antennas can be used in bridge deck surveys. Ground-coupled systems can provide very detailed information about the bridge deck's structures and reinforcement bars. The major problems are the slow speed of data collection and that bridge lanes have to be closed during the data collection. This is why the use of air-coupled antenna systems, which can perform data collection without causing major traffic problems, is highly recommended, especially on high traffic volume roads.

In a bridge deck survey, high frequencies are needed to achieve high resolution as required by identification of small anomalies. As propagation velocity of electromagnetic waves in concrete varies typically from $0.1 \mathrm{~m} / \mathrm{ns}$ to $0.12 \mathrm{~m} / \mathrm{ns}$, using a GPR with the standard frequency $f=1.5 \mathrm{GHz}$ enables theoretically a vertical resolution of at least $40 \mathrm{~mm}$, while $f=2 \mathrm{GHz}$ yields a resolution exceeding the range of $30 \mathrm{~mm}$ [9]. Detecting rebar corrosion in a bridge deck was performed successfully by GPR having a ground coupled antenna working at the frequency of $1.5 \mathrm{GHz}$ [8]. Another study conducted on a bridge deck in Finland demonstrated that the use of a GPR antenna with a frequency of $1 \mathrm{GHz}$ resulted in good radar images [4].

In this work, inspection is considered for reinforced concrete bridge deck overlaid with asphalt by the input reflection coefficient. This consists of a global coefficient resulting from complex sequences of interactions, involving multiple scatterings from the rebar-concrete and the asphalt-concrete interfaces and also from corroded zones in rebars or other defects taken inside the concrete. Focus is on bridge deck suffering from a corroded rebar with various extents. A simplified modelling of corrosion gain is considered here for the reflection from the corroded rebar. A parametric study is conducted after that in order to determine under which circumstances it is possible to operate detection of a corrosion band that is present at the surface of a rebar buried in concrete.

\section{Materials and methods}

GPR can detect echoes from all three types of electrical parameters contrasts: the permittivity $\varepsilon$ in $F / m$, the permeability $\mu$ in $H / m$ and the conductivity $\sigma$ in $\Omega / m$. The permeability is normalized with respect to the free space permeability: $\mu_{0}=4 \pi \times 10^{-7} H / m$, such as $\mu=\mu_{r} \mu_{0}$, while the permittivity is normalized with respect to the permittivity of free space $\varepsilon_{0}=8.8541878176 \times 10^{-12} \mathrm{~F} / \mathrm{m}$ and the relative permittivity as: $\varepsilon_{r}=\varepsilon / \varepsilon_{0}$. From wave propagation point of view, it is motivating to introduce complex relative dielectric constant under the form

$$
\varepsilon_{r}=\varepsilon_{r}^{\prime}-j \varepsilon_{r}^{\prime \prime}
$$

where $\varepsilon_{r}^{\prime}$ is the real part of the complex permittivity, $\varepsilon_{r}^{\prime \prime}$ is the imaginary part of the complex permittivity and $j=\sqrt{-1}$. In general, the real part is responsible for the velocity with which waves travel, while the imaginary part controls the attenuation experienced by the wave.

Under harmonic excitation at frequency $f$, the relative dielectric constant writes

$$
\varepsilon_{r}=\varepsilon_{r}^{\prime}-j \frac{\sigma}{2 \pi f \varepsilon_{0}}
$$

The amplitude and polarity of the wave reflected from a change in dielectric properties depend on the ratio of the two relative dielectric constants and is referred to as the reflection coefficient. Assuming a plane wave normally incident from medium 1 to 2 , the reflection coefficient $R_{1,2}$ can be expressed as follow

$$
R_{1,2}=\frac{\sqrt{\mu_{r 1} \varepsilon_{r 2}}-\sqrt{\mu_{r 2} \varepsilon_{r 1}}}{\sqrt{\mu_{r 1} \varepsilon_{r 2}}+\sqrt{\mu_{r 2} \varepsilon_{r 1}}}
$$

When the magnetic permeabilities of the two materials are equal to that of free space, Equation (3) can be expressed in term of only the dielectric constants of the top (1) and bottom (2) media as

$$
R_{1,2}=\frac{\sqrt{\varepsilon_{r 2}}-\sqrt{\varepsilon_{r 1}}}{\sqrt{\varepsilon_{r 2}}+\sqrt{\varepsilon_{r 1}}}
$$

The magnitude of the reflection coefficient dictates the amplitude of the reflected wave, and the sign of the reflection coefficient dictates the phase shift of the returning wave. Knowing the common values of concrete dielectric properties, reflection occurring on carbon steel is almost quasi-total: $R_{1,2} \simeq 90 \%$.

From Equation (3), it is possible to obtain the power reflection coefficient as

$$
\operatorname{Pr}=\left(R_{1,2}\right)^{2}=\left(\frac{\sqrt{\mu_{r 1} \varepsilon_{r 2}}-\sqrt{\mu_{r 2} \varepsilon_{r 1}}}{\sqrt{\mu_{r 1} \varepsilon_{r 2}}+\sqrt{\mu_{r 2} \varepsilon_{r 1}}}\right)^{2}
$$

Air-coupled GPR produces more difficult data to work with as transmission is poor, but the antennas are not in contact with the ground and data can be collected at a much higher speed without damaging the antennas. For air-coupled GPR, the problem of bridge deck made from reinforced concrete and overlaid with asphalt can be assumed to involve a stratified medium consisting of $N$ homogeneous layers. The upper layer boarding the deck upper side is air and the layer at the bottom side is 
associated to steel rebar level which can be supposed to be a perfectly reflecting surface. An analytic formulation of the reflection and transmission coefficients can be developed by considering the bridge deck configuration geometry depicted in Figure 1. A uniform plane wave with either TE or TM polarisation is emitted by a horn like antenna located at the distance $d_{0}$ from the deck surface called the leading interface which serves as the reference for the reflection and transmission coefficients.

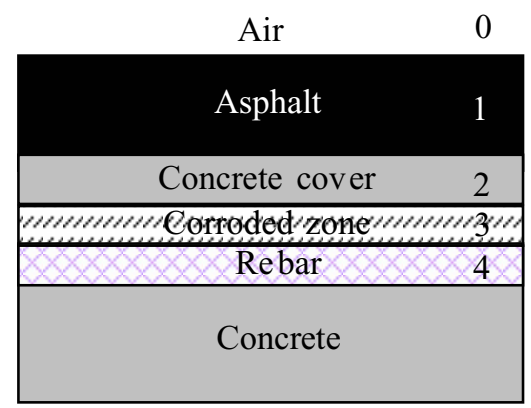

Figure 1. Bridge deck layers with perfect reflecting rebar layer.

The overall reflection and transmission coefficients for perpendicular polarization can be determined recursively [10]. Considering the layers described in Figure 1 under normal incidence, then the value of $\mathrm{N}=3$ and the corroded zone is labelled 3. For this particular situation, one obtains the input reflection coefficient according to the following four recursive steps:

$$
\begin{aligned}
& Y_{4}=\sqrt{\frac{\varepsilon_{4} \mu_{3}\left(1-j \tan \delta_{4}\right)}{\varepsilon_{3} \mu_{4}\left(1-j \tan \delta_{3}\right)}} \\
& A_{3}=\frac{\exp \left(d_{3} \gamma_{3}\right)\left(1-Y_{4}\right)}{2}, B_{3}=\frac{\exp \left(-d_{3} \gamma_{3}\right)\left(1+Y_{4}\right)}{2} \\
& Y_{3}=\sqrt{\frac{\varepsilon_{3} \mu_{2}\left(1-j \tan \delta_{3}\right)}{\varepsilon_{2} \mu_{3}\left(1-j \tan \delta_{2}\right)}} \\
& A_{2}=\frac{\exp \left(d_{2} \gamma_{2}\right)}{2}\left[A_{3}\left(1+Y_{3}\right)+B_{3}\left(1-Y_{3}\right)\right] \\
& B_{2}=\frac{\exp \left(-d_{2} \gamma_{2}\right)}{2}\left[A_{3}\left(1-Y_{3}\right)+B_{3}\left(1+Y_{3}\right)\right] \\
& Y_{2}=\sqrt{\frac{\varepsilon_{2} \mu_{1}\left(1-j \tan \delta_{2}\right)}{\varepsilon_{1} \mu_{2}\left(1-j \tan \delta_{1}\right)}} \\
& A_{1}=\frac{\exp \left(d_{1} \gamma_{1}\right)}{2}\left[A_{2}\left(1+Y_{2}\right)+B_{2}\left(1-Y_{2}\right)\right] \\
& B_{1}=\frac{\exp \left(-d_{1} \gamma_{1}\right)}{2}\left[A_{2}\left(1-Y_{2}\right)+B_{2}\left(1+Y_{2}\right)\right] \\
& Y_{1}=\sqrt{\frac{\varepsilon_{1} \mu_{2}\left(1-j \tan \delta_{1}\right)}{\varepsilon_{0} \mu_{1}}}
\end{aligned}
$$

$$
\Gamma_{i n}=\exp \left(-2 d_{0} \gamma_{0}\right) \frac{A_{1}\left(1-Y_{1}\right)+B_{1}\left(1+Y_{1}\right)}{A_{1}\left(1+Y_{1}\right)+B_{1}\left(1-Y_{1}\right)}
$$

with for $n=1, \ldots, 4$

$$
\gamma_{n}=2 \pi f \sqrt{-\mu_{n} \varepsilon_{n}+j \frac{\sigma_{n} \mu_{n}}{2 \pi f}} \text { and } \tan \delta_{n}=\frac{\sigma_{n}}{2 \pi f \varepsilon_{n}^{\prime}}
$$

where $d_{n}$ is the thickness of layer number $n, \Gamma_{i n}$ is the input reflection coefficient and $T_{i n}$ is the input transmission coefficient.

The reflection coefficient $\Gamma_{\text {in }}$ contains information on the layers crossed by the electromagnetic wave. It depends in particular on the thickness of the corroded zone. The presence of corrosion modifies the input reflection coefficient $\Gamma_{i n}$ through the coefficients $A_{3}, B_{3}, Y_{3}$ and $Y_{4}$.

In the absence of corrosion, the layer number three vanishes and the recursive steps are modified according to

$$
\begin{gathered}
Y_{4}=\sqrt{\frac{\varepsilon_{4} \mu_{2}\left(1-j \tan \delta_{4}\right)}{\varepsilon_{2} \mu_{4}\left(1-j \tan \delta_{2}\right)}} \\
A_{2}=\frac{\exp \left(d_{2} \gamma_{2}\right)\left(1-Y_{4}\right)}{2}, B_{2}=\frac{\exp \left(-d_{2} \gamma_{2}\right)\left(1+Y_{4}\right)}{2} \\
Y_{2}=\sqrt{\frac{\varepsilon_{2} \mu_{1}\left(1-j \tan \delta_{2}\right)}{\varepsilon_{1} \mu_{2}\left(1-j \tan \delta_{1}\right)}}
\end{gathered}
$$

with the last step (last four lines) remaining the same as in equation (6).

\section{Results and discussion}

In order to assess the effect of corrosion, a Matlab script was developed for both corrosion being present in the bridge deck, equation (6), and corrosion free situation, equation (7). Comparison was then made between these two conditions as function of wave frequency.

A bridge deck having the form given in Figure 1 was considered. The problem parameters are given in Table 1 , where some conductivies are function of the wave frequency. The investigated interval of frequencies was fixed to be $[1,2] \mathrm{GHz}$ and corrosion zone thickness was taken in the interval $[0,5] \mathrm{mm}$. Figure 2 gives the evolution of the input reflexion coefficient as function of the work frequency for the corrosion free bridge deck an three different values of the corrosion layer thickness.

Figure 3 gives the input reflexion coefficient as function of the corroded layer thickness for various values of the work frequency. Note that there are intersection between the curve $x=0$ which corresponds to a corrosion free bridge deck and any other amount of corrosion thickness. This occurs for a specific frequency.

Figure 2 shows that, for a given thickness of the corrosion layer, there are frequencies for which no distinction can be made in terms of the input reflection coefficient between corroded rebar layer and corrosion 
free bridge deck. Figure 3 shows that for $f=1.75 \mathrm{GHz}$ and for two different corrosion levels the input reflexion coefficient has the same value. This signifies that estimation of the amount of corrosion cannot be made by working with this frequency.

Table 1. Geometric and dielectric properties of the bridge deck layers as function of the work frequency $f$ and corrosion layer thickness $x$.

\begin{tabular}{|c|c|c|c|c|}
\hline Layer & $d(\mathrm{~cm})$ & $\mu_{r}$ & $\varepsilon_{r}^{\prime}$ & $\sigma(S / m)$ \\
\hline Air & 1 & 1 & 1 & 0 \\
\hline Asphalt & 2 & 1 & 4.18 & 0 \\
\hline Concrete & 3 & 1 & 6.20 & $2 \times 10^{-11} \times f$ \\
\hline Corrosion & $x$ & $2-0.3 j$ & 1.5 & $5.56 \times 10^{-12} \times f$ \\
\hline Rebar & $2.5-x$ & 100 & 1.45 & $9.931 \times 10^{6}$ \\
\hline
\end{tabular}

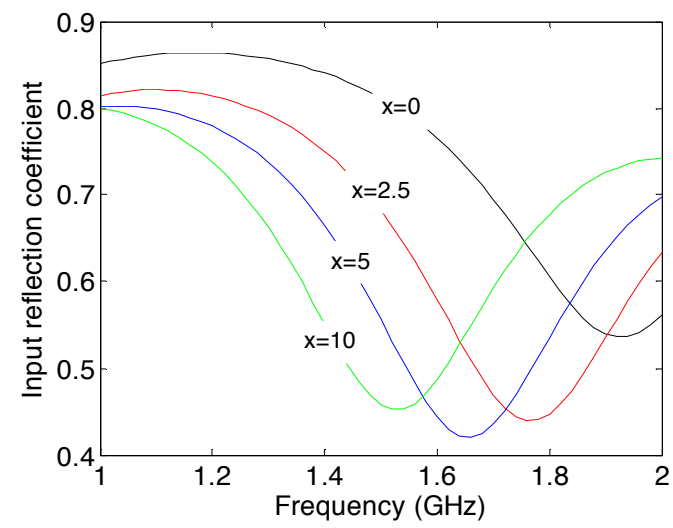

Figure 2. Input reflection coefficient as function of the harmonic wave frequency for various corrosion layer thicknesses.

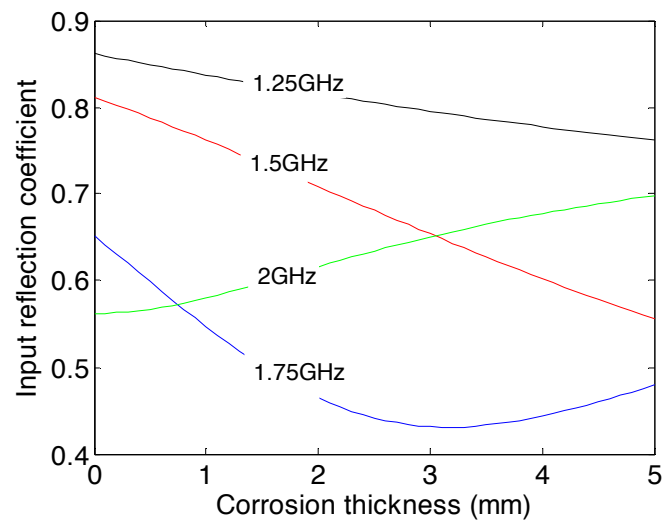

Figure 3. Input reflection coefficient as function of the corrosion layer thickness for various harmonic wave frequencies.
The obtained results emphasize the fact that for operating corrosion detection in bridge deck, one has to choose the right frequency, otherwise corrosion can be masked or corrosion extent cannot be estimated. As the bridge deck configuration is variable, it is recommended in practice to use a packet of frequencies.

\section{Conclusions}

A methodological approach for the detection of rebar corrosion anomaly in bridge deck made from reinforced concrete was presented. This methodology employs GPR acquired data in terms of the overall input reflection coefficient when perpendicular polarization of the harmonic electromagnetic wave is used under normal incidence. It was found that the input reflection coefficient depends hugely on the frequency of harmonic wave. As selection of the wave that enables to monitor corrosion depends on the actual problem, it is recommended then to use a packet of waves centred on a given high frequency. This will increase resolution and corrosion detection. Experimental validation is needed in order to assess these findings.

\section{References}

1. H. M. Jol, Ground Penetrating Radar: Theory and Applications (Elsevier, Amsterdam, 2008)

2. J.H. Bungey, Constr. Build. Mater. 18 (2004)

3. V. Barrile, R. Pucinotti, NDT\&E International 38 (2005)

4. N. Parry, J.L. Davis, Geological Survey of Finland 16 (1992)

5. T. Alongi, C.G. Clemena, P.D. Cady, Condition Evaluation of Concrete Bridges relative to Reinforcement Corrosion (SHRP Report, Washington, DC, 1992)

6. I. Al-Qadi, K. Jiang, S. Lahouar, Bridge deck reinforcing steel cover depth prediction using GPR (Transportation Research Board 85th Annual Meeting, Washington, 2006)

7. K.R. Maser, K. Tabrizi, V. Gangi, Use of Nondestructive Methods for Large Scale Bridge Deck Evaluation (TRB, National Research Council, Washington, 2001)

8. S. Laurens, J.P. Balayssac, J. Rhazi, G. Klysz, G. Arliguie, Materials and Structures 38 (2005)

9. C.W. Chang, C.H. Lin, H.S. Lien, Constr Build Mater, 23 (2009)

10. C.A. Balanis, Advanced Engineering Electromagnetics (Wiley, New York, 1989) 